\title{
Electrostatic-Spray Ionization Mass Spectrometry
}

\author{
Liang Qiao, ${ }^{\dagger, \S}$ Romain Sartor, ${ }^{\dagger, \S}$ Natalia Gasilova, ${ }^{\dagger}$ Yu Lu, ${ }^{\dagger}$ Elena Tobolkina, ${ }^{\dagger}$ Baohong Liu, ${ }^{\ddagger}$ \\ and Hubert H. Girault*, ${ }^{\dagger}$ \\ ${ }^{\dagger}$ Laboratoire d’Electrochimie Physique et Analytique, Ecole Polytechnique Fédérale de Lausanne, Station 6, CH-1015 Lausanne, \\ Switzerland \\ ${ }^{\ddagger}$ Department of Chemistry, Institute of Biomedical Sciences, Fudan University, Shanghai, 200433, P.R. China
}

\section{Supporting Information}

ABSTRACT: An electrostatic-spray ionization (ESTASI) method has been used for mass spectrometry (MS) analysis of samples deposited in or on an insulating substrate. The ionization is induced by a capacitive coupling between an electrode and the sample. In practice, a metallic electrode is placed close to but not in direct contact with the sample. Upon application of a high voltage pulse to the electrode, an electrostatic charging of the sample occurs leading to a bipolar spray pulse. When the voltage is positive, the bipolar spray pulse consists first of cations and then of anions. This method has been applied to a wide range of geometries to emit ions from samples in a silica capillary, in a disposable pipet tip, in a polymer
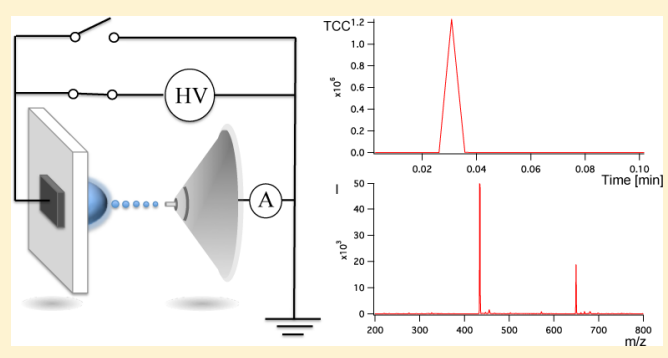
microchannel, or from samples deposited as droplets on a polymer plate. Fractions from capillary electrophoresis were collected on a polymer plate for ESTASI MS analysis.

$\mathrm{E}$ lectrospray is a phenomenon studied as early as 1749 when Nollet described the spray from a metallic orifice that was electrostatically electrified. ${ }^{1}$ Since the 1980 s, electrospray ionization (ESI) has been widely used as a powerful technique to softly ionize large compounds from solution for mass spectrometry (MS) analysis. ${ }^{2-4}$ Nowadays, ESI is a general ionization technique that has been applied to a wide range of biomolecules and coupled with various types of mass analyzers, including ion traps (IT), time-of-flight (TOF), quadrupole, Fourier-transform ion cyclotron resonance (FTICR), and ITorbitrap. $^{5-8}$

ESI is based on the ejection of charged droplets from the tip of a capillary or a microchannel. The droplets reduce in size during the flight by solvent evaporation and/or by Coulomb explosion to form gas phase ions representative of the species in solution. ${ }^{9}$ In traditional ESI-MS, a direct current (DC) high voltage is applied between an electrode contacting the solution in a microchannel or a capillary and the mass spectrometer acting as the counter electrode. When a current flows through the electrospray emitter, electrochemical reactions occur at the electrodelsolution interface to ensure the electroneutrality of the solution and to form charged droplets for ESI. ${ }^{9-11}$

Contactless electrochemical techniques based on capacitive coupling have been developed either to monitor the adsorption of molecules on a substrate by measuring variations of interfacial capacitances ${ }^{12}$ or to measure the conductivity of an electrolyte solution within an insulating container. The latter is routinely used to detect chemicals fractionated by capillary electrophoresis (CE). ${ }^{13-15}$

Recently, Cooks et al. have reported a contactless method to generate a pulsed spray. ${ }^{16,17} \mathrm{~A}$ pulsed high voltage waveform was applied on an electrode $2 \mathrm{~mm}$ away from a nanospray emitter to induce voltage inside the emitter. Similarly, Zhang et al. have used alternating current (AC) high voltages to perform a contactless spray, where the charge states of peptides were controllable. $^{18}$ In comparison with classical ESI, the high voltage was not directly applied to the sample solution, and no electrode reaction could occur. This is an advantage when electrochemical reactions during conventional ESI need to be avoided. Even in a contact mode, pulsed spray sources were found to be useful when mass spectrometry was coupled with microseparation strategies for analyzing trace amounts of a sample mixture. ${ }^{19-22}$ The former publications about contactless spray were mainly focused on applications. A comprehensive investigation on the working mechanisms is of interest for development of new techniques and for opening a wide range of applications for mass spectrometry analysis.

Herein, we employed a constant high voltage power supply and an electrical circuit comprising two synchronized switches to generate high voltage pulses for onsetting a spray. The principle of this contactless spray was thoroughly investigated and shown to stem from a capacitive coupling effect. Under the application of a high voltage on the electrode isolated from sample solution with respect to the MS at ground, two capacitors are formed in series: electrode-insulator-sample solution and sample solution-gas-MS inlet. During electrostatic charging of the capacitors under high voltage, charges accumulate at the solution-gas interface. When the electrostatic pressure is larger than the Laplace pressure, spray

Received: May 16, 2012

Accepted: August 9, 2012

Published: August 9, 2012 
Scheme 1. Schematic Representation of the Setups for Electrostatic-Spray Ionization of Solutions from (a) a Microfluidic Chip and (b) a Micropipet Tip; (c) Schematic Representation of the Electrostatic-Spray Ionization of Samples in a Micro-Droplet on an Insulating Plate ${ }^{a}$

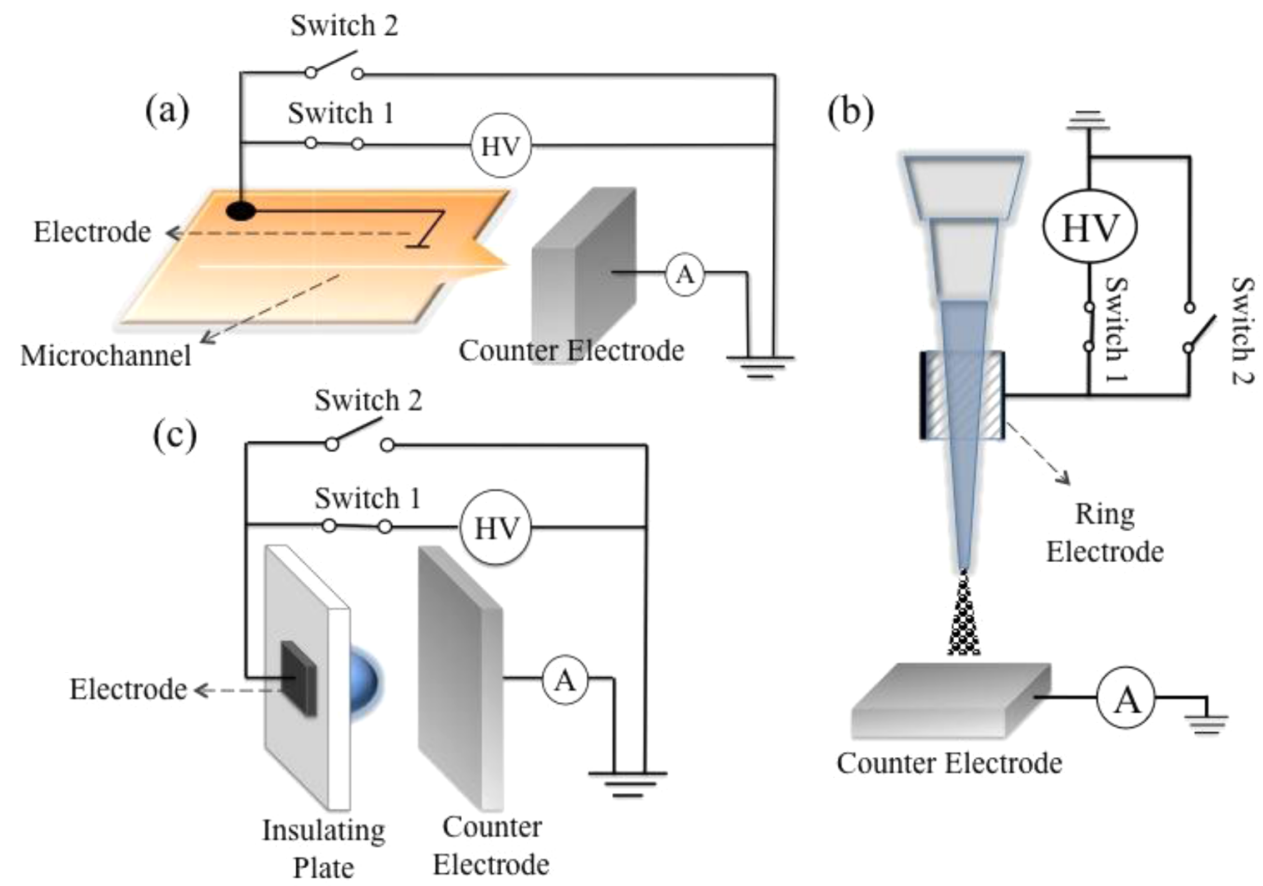

${ }^{a}$ The counter electrode can be either a mass spectrometer or simply a metallic plate. HV: high voltage; A: ammeter.

ionization happens. By grounding the electrode, capacitors are discharged and spray of ions with opposite charges happens. On the basis of this electrostatic capacitor chargingdischarging principle, we named the contactless spray technique as electrostatic-spray ionization (ESTASI). We have tested this ESTASI approach on different systems such as microfluidic chips, micropipet tips, and silica capillaries. Furthermore, the spray has been directly performed from microdroplets on an insulating plate, making spray ionization of the samples possible from any substrates. We have collected fractions from capillary electrophoresis (CE) as microdroplets on an insulating plate for capacitive ESTASI MS analysis. ESTASI should open a wide range of applications for mass spectrometry analysis.

\section{EXPERIMENTAL SECTION}

Information on chemicals and fabrication of microchip is given in Supporting Information SI-1.

Electrostatic-Spray Ionization. When spraying from a microchannel in a microfluidic chip or from a polyimide coated silica capillary, an electrode was placed close to the microchannel or capillary. A high potential $( \pm 6 \mathrm{kV})$ was applied to the electrode by closing switch 1 , switch 2 being open as shown in Scheme 1. The electrode was then grounded after opening switch 1 and closing switch 2 . A LabView program was written to control the two switches to synchronize their operation. The end of the microchannel was placed just in front of the mass spectrometer inlet. A syringe pump was used to infuse a sample solution into the microchannel at adjustable flow rates. Warning: It is important to use proper isolation measures when pulsing high voltages as electromagnetic spikes are generated.

To spray from a micropipet tip, $1 \mu \mathrm{L}$ of sample solution was previously loaded into the disposable tip. An electrode was placed around the tip. By applying a high potential pulse on the electrode using the two switches, ESTASI was realized.

To spray from microdroplets, droplets of the sample solution (1 $\mu \mathrm{L})$ were deposited on an poly(methylmethacrylate) (PMMA) plate patterned with wells or hydrophilic patches. The electrode was placed behind the plate close to the droplet to induce the spray. The droplet was put in front of the mass spectrometer inlet. By applying high potential pulses to the electrode using the two switches, ESTASI was generated.

CE Coupled with ESTASI MS. A mixture of peptides generated from the tryptic digestion of myoglobin was used as sample for CE separation coupled with ESTASI. The digestion was performed by incubating $5 \mathrm{mg} / \mathrm{mL}$ myoglobin (horse heart) with $0.17 \mathrm{mg} / \mathrm{mL}$ trypsin (bovine pancreas) in a buffer of $25 \mathrm{mM} \mathrm{NH}_{4} \mathrm{HCO}_{3}(1 \mathrm{~mL})$ under $37{ }^{\circ} \mathrm{C}$ for $18 \mathrm{~h}$. The protein was denatured by heating at $100{ }^{\circ} \mathrm{C}$ for $5 \mathrm{~min}$ prior to tryptic digestion.

Standard CE separation of the myoglobin tryptic digest (150 $\mu \mathrm{M}, 21 \mathrm{~nL}$ per sample injection) followed with UV detection was first performed on an Agilent 7100 CE system (Agilent, Waldbronn, Germany). An untreated fused silica capillary (50 $\mu \mathrm{m}$ inner diameter, $375 \mu \mathrm{m}$ outside diameter, $51.5 \mathrm{~cm}$ effective length, $60 \mathrm{~cm}$ total length) obtained from BGB analytik AG (Böckten, Switzerland) was used for separation. Solution of $10 \%$ acetic acid, $\mathrm{pH}=2$, has been employed as a background electrolyte. Sample was injected during $20 \mathrm{~s}$ at pressure of 42 mbar. Separation was performed at a constant voltage of $30 \mathrm{kV}$.

Afterward, the capillary was cut at the point of detection window and then coated with a conductive silver ink (Ercon, Wareham, MA, USA) over a length of $10 \mathrm{~cm}$ from the outlet that was then fixed outside the CE apparatus. The same CE separation was performed with the same sample, while the fractions were directly collected on an insulating polymer plate by a homemade robotic system that was previously designed for 
Scheme 2. Equivalent Circuit of the Electrostatic-Spray Ionization during Capacitors (a) Charging and (b) Discharging ${ }^{a}$

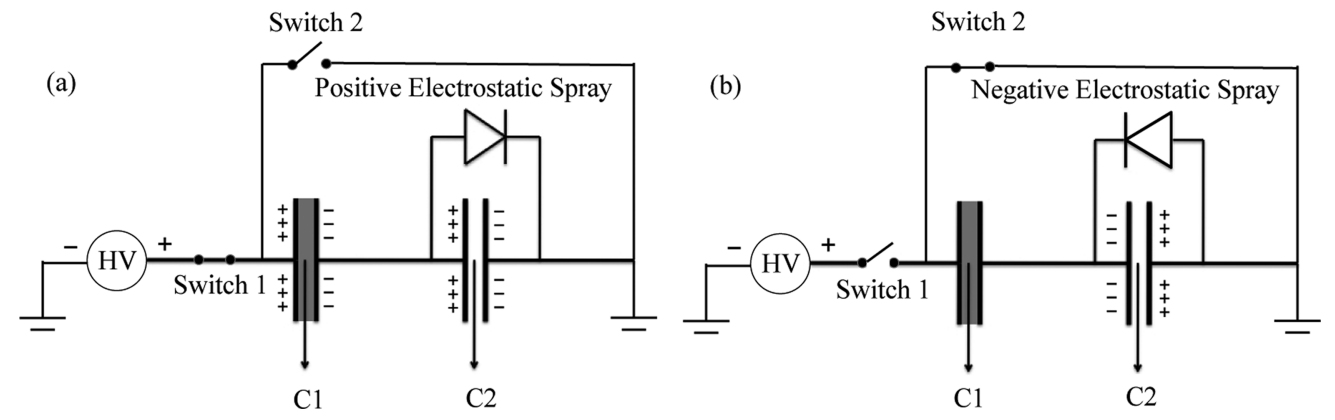

${ }^{a}$ The diode symbolizes the direction of the spray current: spray of cations in (a) and spray of anions in (b).

collecting CE fractions on a matrix-assisted laser desorption/ ionization (MALDI) sample plate. $^{23}$ Silver ink coating was connected to the ground during CE separation.

After drying all the droplets, the polymer plate was placed between the electrode and MS inlet. One microliter of an acidic buffer (1\% acetic acid in 49\% water and 50\% methanol $(\mathrm{MeOH})$ ) was deposited on each sample spot to dissolve the peptides for ESTASI-MS detection.

For comparison, a parallel experiment was performed to collect fractions from CE separation on a MALDI sample plate. The fractions were then analyzed by a Bruker microflex MALDI-TOF mass spectrometer (Bruker Daltonics, Bremen, Germany).

Mass Spectrometer. The ESI and ESTASI-MS experiments were performed on a Thermo LTQ Velos mass spectrometer. An enhanced trap scan rate $(10000 \mathrm{Th} / \mathrm{s})$ was selected to obtain a good spectral resolution. During ESTASI, the spray voltage of the internal power source of the LTQ Velos was set to 0 . A battery that can provide a high voltage up to $10 \mathrm{kV}$ was used as the external power source to induce the spray.

The MALDI-MS experiments were performed on a Bruker microflex MALDI-TOF mass spectrometer. After drying the fractions from $\mathrm{CE}, 1 \mu \mathrm{L}$ of $10 \mathrm{mg} / \mathrm{mL}$ 2,5-dihydroxybenzoic acid in $50 \%$ water and $50 \%$ acetonitrile was deposited on each sample spot as matrix. The mass spectrometer worked under the reflectron-scanning mode for all experiments.

\section{RESULTS AND DISCUSSION}

ESTASI Principle. The ESTASI principle is demonstrated here using a microfluidic chip consisting of a microchannel $(100 \mu \mathrm{m} \times 50 \mu \mathrm{m})$ and an electrode as shown in Scheme 1a. The electrode was made with carbon ink and placed $2 \mathrm{~mm}$ away from the microchannel as a band having a lateral surface area of $50 \mu \mathrm{m} \times 5 \mathrm{~mm}$. Either the mass spectrometer or a metallic plate was used as the counter electrode. The metallic plate was used for measuring the current transients.

When the electrode is connected to the high potential source (switch 1 closed, switch 2 open), the system behaves as two capacitors in series before the electrospray happens. The first capacitor (C1) is a conductor (the electrode)-insulator-liquid solution capacitor, and no current can flow through it; its capacitance depends on the geometry of the device. The second capacitor (C2) is at the spray emitter and is a liquid solutiongas-metal (the counter electrode or mass spectrometer) capacitor; its capacitance varies as a function of the position of the emitter with respect to the mass spectrometer. When the charge accumulated on the second capacitor is too large, the surface tension at the tip is not sufficient to prevent the emission of charged droplets, and this second capacitor can be considered as a leaky capacitor with a diode in parallel, Scheme $2 a$.

Indeed, electrospray occurs when the electrostatic pressure at the tip of the emitter is larger than the Laplace pressure. The electrostatic pressure is a function of the surface charge accumulation

$$
p_{\mathrm{E}}=\sigma^{2} / 2 \varepsilon_{0}
$$

where $\sigma$ is the surface charge density and $\varepsilon_{0}$ is the permittivity of vacuum. If the tip of the emitter, e.g., at the Taylor cone, is assumed to be hemispherical, the Laplace pressure is simply given by

$$
P_{\mathrm{L}}=2 \gamma / r
$$

where $\gamma$ is the surface tension of the liquid and $r$ is the radius of the hemisphere from where droplets are formed. To obtain electrospray, it is important to keep the surface of the solution small enough to increase $\sigma$ for a given interfacial charge stored on the capacitor $\mathrm{C} 2$.

The equivalent capacitance of the whole system is given as: $C_{\mathrm{eq}}=C_{1} C_{2} /\left(C_{1}+C_{2}\right)$, where $C$ is the capacitance of the corresponding capacitor $C$. Upon application of a high voltage $U$, the maximum charge on the second capacitor representing the tip of the emitter is $U C_{\text {eq }}$. By placing the emitter far from the MS inlet, $C_{2}$ becomes very small. $C_{\mathrm{eq}}$ is then equal to $C_{2}$, and the maximum charge at the tip of the emitter is $U C_{2}$. Since $\mathrm{C}_{2}$ is very small, this charge is too small to induce electrospray. By placing the emitter close enough to MS inlet, $C_{2}$ cannot be neglected, and the charge at the tip is $U C_{\text {eq }}$. When this charge is large enough, the emitter will spray.

In the case of the microchip shown in Scheme 1a, the first capacitor can be approximately viewed as a parallel-plate capacitor, and the capacitance is

$$
C_{1}=\varepsilon_{0} \varepsilon_{\mathrm{r}} A / d
$$

where $A$ is the lateral surface area of the electrode $(50 \mu \mathrm{m} \times 5$ $\mathrm{mm}), d$ is the distance between the band electrode and the microchannel $(2 \mathrm{~mm})$, and $\varepsilon_{\mathrm{r}}$ is the relative permittivity of the insulator, here 3.5 for PI. Therefore, to facilitate ESTASI, one should minimize $d$ and maximize $A$. Specifically, with the geometry of the microchip illustrated in Scheme la, the capacitance of the first capacitor is around $4 \mathrm{fF}$, which could supply a charge around $20 \mathrm{pC}$ when applying a high voltage of $6 \mathrm{kV}$, which is enough to onset the spray as detailed in the Supporting Information SI-2. These data should be compared with ESI from a similar microchip but where the electrode is in 
contact with the solution. Typically, during microchip ESI, we operate with a high voltage of $4 \mathrm{kV}$ and a total ion current of $100 \mathrm{nA}$, which yields a resistance value for the air gap of $40 \mathrm{G} \Omega$.

As the spray occurs, the second capacitor leaks and is bypassed by a resistance $(R)$ and the system behaves as a $R C$ circuit, with a time constant $\tau=R C_{1}$, i.e., around $0.2 \mathrm{~ms}$ for the microchip. The experimental time constant is in the order of $0.1 \mathrm{~s}$ and stems mainly from the rise time of the switch box system. Similar calculations for the other emitters are given in the Supporting Information SI-2.

When part of the positive charge at the tip of the solution is sprayed, the positive charge stays on the electrode, meaning that an excess negative charge builds up in the solution during the spray. To alleviate this problem, the electrode is then disconnected from the power supply and grounded to discharge both capacitors. Since the charge stored in the first capacitor is large enough to induce electrospray, part of the negative charge built up at the surface of the solution can be released by spray of anions, Scheme $2 \mathrm{~b}$. By repeating this cycle, alternating electrostatic-spray of cations and anions is realized during the charging-discharging of the two capacitors.

Ion Current during ESTASI. Current transients generated during ESTASI were measured using a metallic plate counter electrode. ESTASI was realized by pushing a solution of $50 \%$ $\mathrm{MeOH}, 49 \% \mathrm{H}_{2} \mathrm{O}$, and $1 \%$ acetic acid through the microchannel in the microchip, shown as Scheme 1a, and applying a high potential pulse on the electrode integrated in the microchip. The metallic plate was placed $0.5 \mathrm{~cm}$ away from the microchip emitter. As shown in Figure 1, when a positive

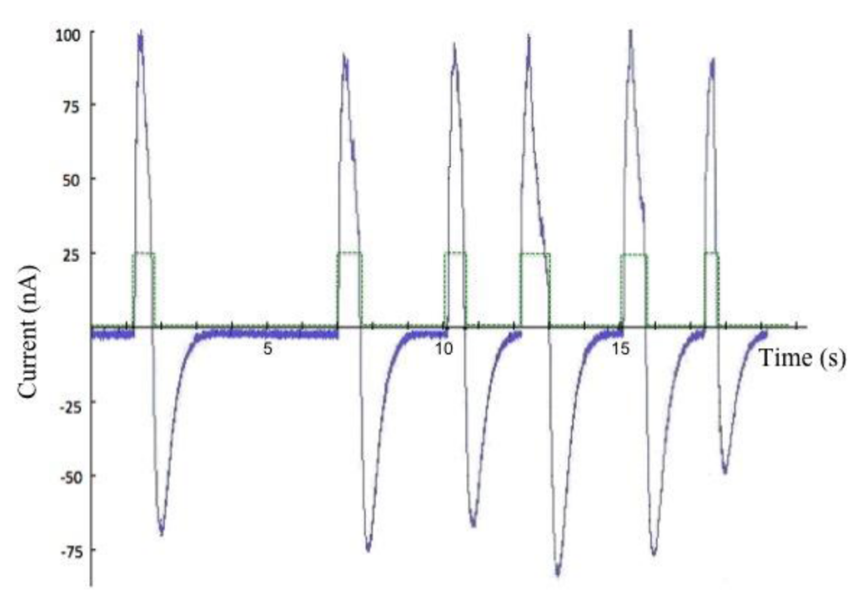

Figure 1. Current transients measured (blue line) using a counter electrode during ESTASI. The application of a high potential pulse is shown as the dashed green line. A solution of $50 \% \mathrm{MeOH} / 49 \% \mathrm{H}_{2} \mathrm{O}$ / $1 \%$ acetic acid was used for the electrostatic-spray. A high potential of $6 \mathrm{kV}$ was applied on the electrode to induce the electrospray.

high voltage was applied, a positive spray current with a peak value of around $100 \mathrm{nA}$ was observed. A negative spray current was detected as soon as the electrode was disconnected from the power supply and grounded. The current transients observed corroborate directly the capacitor charging-discharging principle of ESTASI.

ESTASI-MS from a Microchip: Standard Samples, Limit of Detection, and Large Proteins. When a mass spectrometer was used as the counter electrode, mass-to-charge $(\mathrm{m} / \mathrm{z})$ ratios of the ions generated from ESTASI were analyzed. Angiotensin I solution $\left(0.1 \mathrm{mM}\right.$ in $50 \% \mathrm{MeOH} / 49 \% \mathrm{H}_{2} \mathrm{O} / 1 \%$ acetic acid) was infused by a syringe pump into the microchannel of the microfluidic chip to be sprayed and analyzed by MS. The switches were operated by LabView to perform a series of pulses.

ESTASI-MS results shown in Figure 2 were obtained under a flow rate of $20 \mu \mathrm{L} / \mathrm{h}$ and the following time sequence parameters: $t_{1}=t_{4}=0.05 \mathrm{~s}, t_{2}=0.25 \mathrm{~s}$, and $t_{3}=0.15 \mathrm{~s}$. The definitions of $t_{1}, t_{2}, t_{3}$, and $t_{4}$ are shown in Scheme SI-3, Supporting Information. Figure $2 \mathrm{a}$ shows the total cation current (TCC) recorded by the MS as a function of time, which indicates a very stable pulsed ESTASI process. Zooming in at the current signal, we found clearly in Figure $2 b$ that the peaks of TCC appeared periodically every $1.5 \mathrm{~s}$. In each cycle, four mass spectra were collected with similar time intervals, indicating that the generation of each spectrum took $375 \mathrm{~ms}$ and that one spectrum included 1.25 charging-discharging cycles. Mass spectra collected from the peak (Figure 2c) of TCC showed peaks of the doubly and triply protonated angiotensin I. In contrast, a weak signal was generated from the baseline of the TCC, Figure 2d. Each cycle of the MS detection is formed by several procedures, including injection, ion transfer, ion scanning, and ion detection. Only when the electrostatic-spray ionization happens during the injection, an intensive spectrum is obtained, corresponding to the cycle of the TCC as a function of time. Here, the maximum injection time was set to $50 \mathrm{~ms}$.

The analytical figures of merit of ESTASI are further characterized and compared with ESI. We found that peptides or small proteins with a concentration of $50 \mathrm{nM}$ could be efficiently ionized by ESTASI and detected by a LTQ Velos mass spectrometer (Figure 3a,c). The microchip emitter and experimental conditions were the same as those in Figure 2. The limit of detection (LOD) of ESTASI-MS was found to be at 5 to $10 \mathrm{nM}$ for standard peptides and at 10 to $15 \mathrm{nM}$ for standard small proteins (Figure 3b,d). For comparison, ESI was performed with a similar microchip emitter, where the geometry of microchannel is completely the same as the one used for ESTASI while the electrode contacts directly the solution inside the microchannel, shown as Scheme SI-2.1, Supporting Information. The LOD was found to be at 20 to 25 $\mathrm{nM}$ and 45 to $50 \mathrm{nM}$ for the same peptides and proteins, respectively, under the same experimental conditions, Figure SI-4.1, Supporting Information. Actually, the microchip ESI shows slightly better LOD than ESI performed with the standard commercial ionization source. The latter holds LOD for the same peptide and protein of 45 to $50 \mathrm{nM}$ and 65 to 70 $\mathrm{nM}$, respectively (Figure SI-4.2, Supporting Information). Detailed information on LOD characterization is in Supporting Information SI-4. The better LOD of ESTASI compared to ESI may stem from its pulsed spray nature or the higher voltages used.

Large proteins can also be analyzed by ESTASI-MS. Bovine serum albumin with a molecule weight of $66 \mathrm{kDa}$ was well detected by microchip ESTASI-MS, and the peaks for BSA with various charge states were clearly observed, Figure SI-5a, Supporting Information. When bovine lactoferrin $(76 \mathrm{kDa})$ was used, the protein could be observed by ESTASI-MS. However, it was hard to distinguish the ions with different charge states, Figure SI-5b, Supporting Information. Similar results were obtained with ESI-MS under the same experimental conditions for the two large proteins, Figure SI-5c,d, Supporting Information. Despite the ionization method, the mass analyzer (linear ion trap) and detector can also limit the detection of 

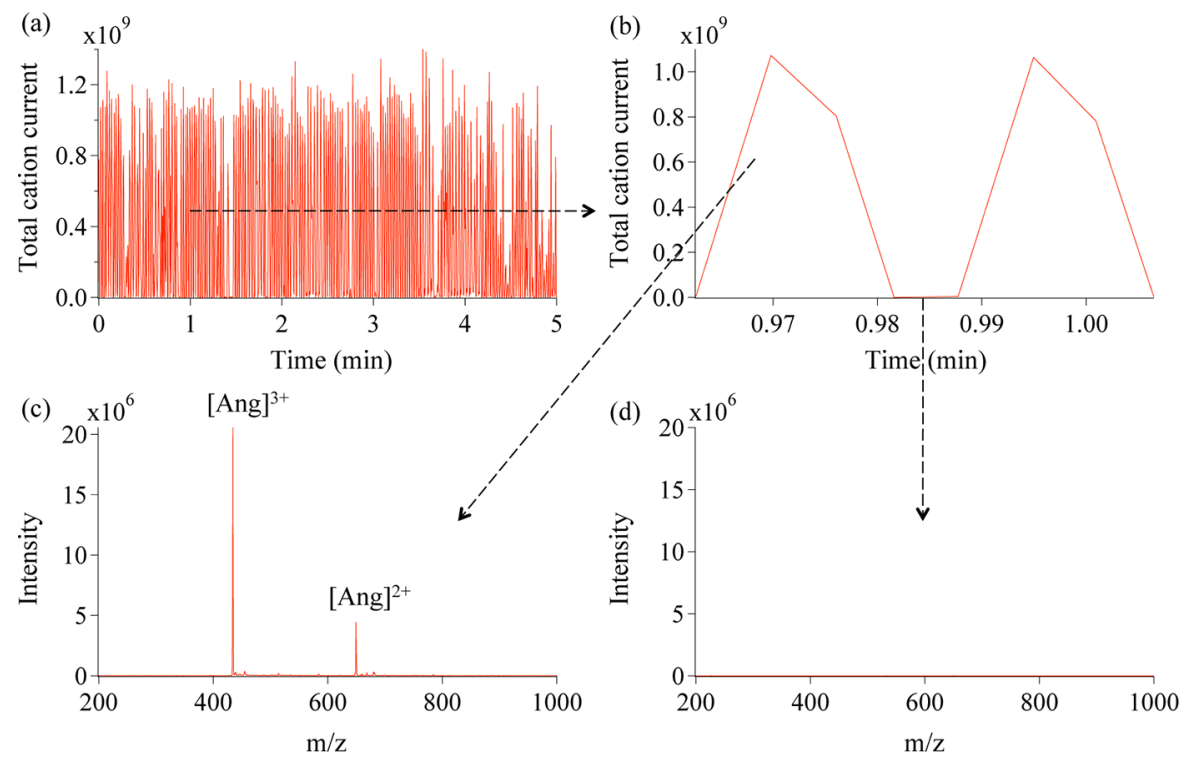

Figure 2. (a, b) Total cation current (TCC) as a function of time and (c, d) the mass spectra under positive MS mode. The ions were generated by electrostatic-spray ionization with a microfluidic chip as emitter when a high potential $(6 \mathrm{kV})$ was applied. The mass spectrometer was scanning under a range of $150 \mathrm{Th}$ to $2000 \mathrm{Th}$ and a scan rate of $10000 \mathrm{Th} / \mathrm{s}$. Only a range from $200 \mathrm{Th}$ to $1000 \mathrm{Th}$ is shown here since the rest is blank without any peaks.
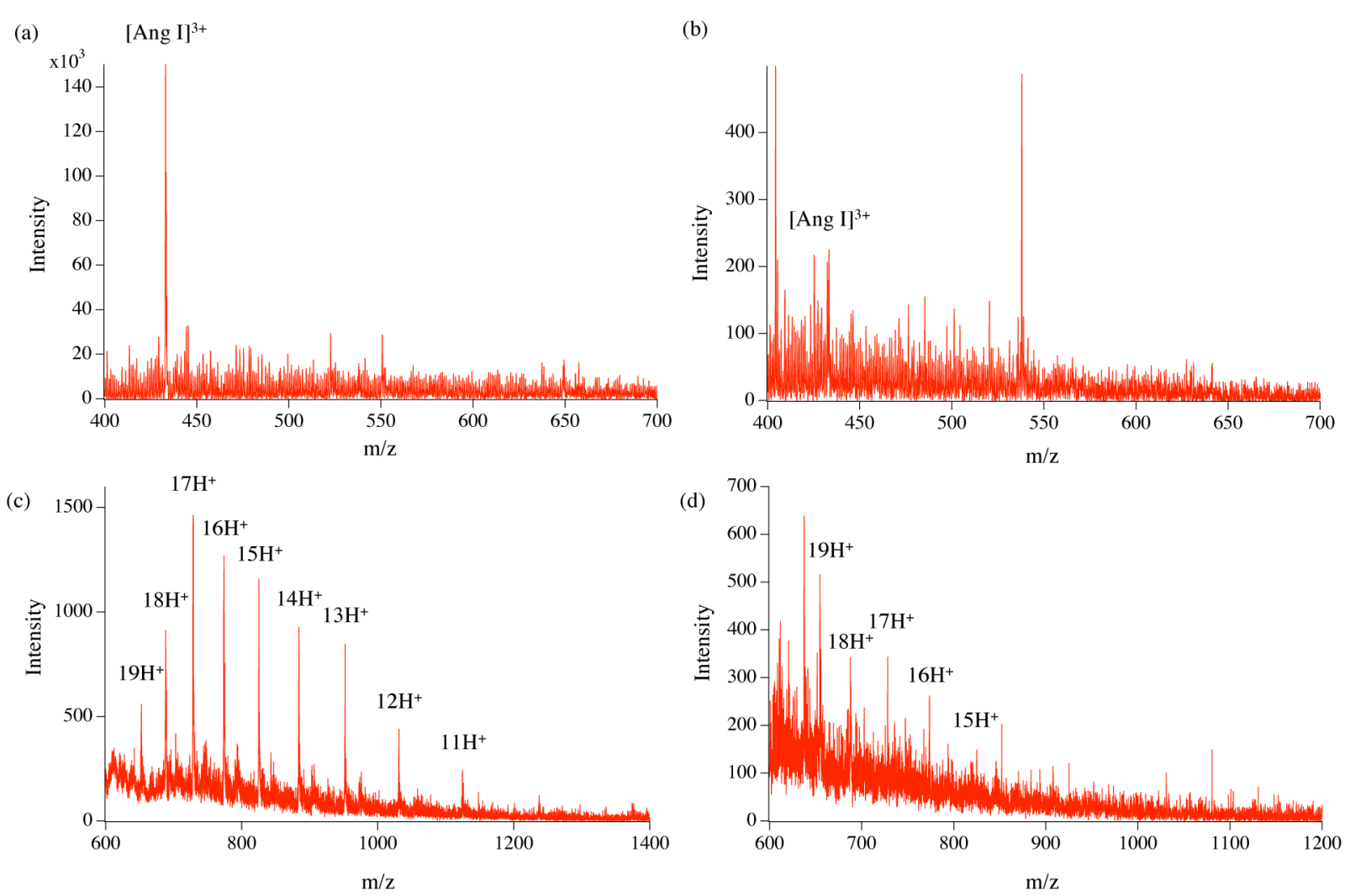

Figure 3. The mass spectra of angiotensin I ((a) $50 \mathrm{nM}$ and (b) $5 \mathrm{nM}$ ) and cytochrome C ((c) $50 \mathrm{nM}$ and (d) $10 \mathrm{nM})$ under positive MS mode. Samples were all prepared in a buffer of $50 \% \mathrm{MeOH} / 49 \% \mathrm{H}_{2} \mathrm{O} / 1 \%$ acetic acid. The ions were generated by ESTASI with a microchip emitter when a high potential $(6 \mathrm{kV})$ was applied. The sample solutions were infused into the microchip by a syringe pump at a flow rate of $20 \mu \mathrm{L} / \mathrm{h}$. Pulse sequence parameters were set to $t_{1}=t_{4}=0.05 \mathrm{~s}, t_{2}=0.25 \mathrm{~s}$, and $t_{3}=0.15 \mathrm{~s}$.

large proteins. These results indicate that ESTASI does not limit the ionization of large proteins up to $76 \mathrm{kDa}$ compared to ESI.

ESTASI-MS from a Silica Capillary: Sequential Spray of

Cations and Anions. ESTASI-MS was also tested with a commercial polyimide coated silica capillary $(50 \mu \mathrm{m}$ inner diameter, $360 \mu \mathrm{m}$ outside diameter), the electrode being merely a steel crocodile clip surrounding the outer surface of the capillary, which can be simply viewed as a ring electrode to generate a cylindrical capacitor with the electrolyte solution 

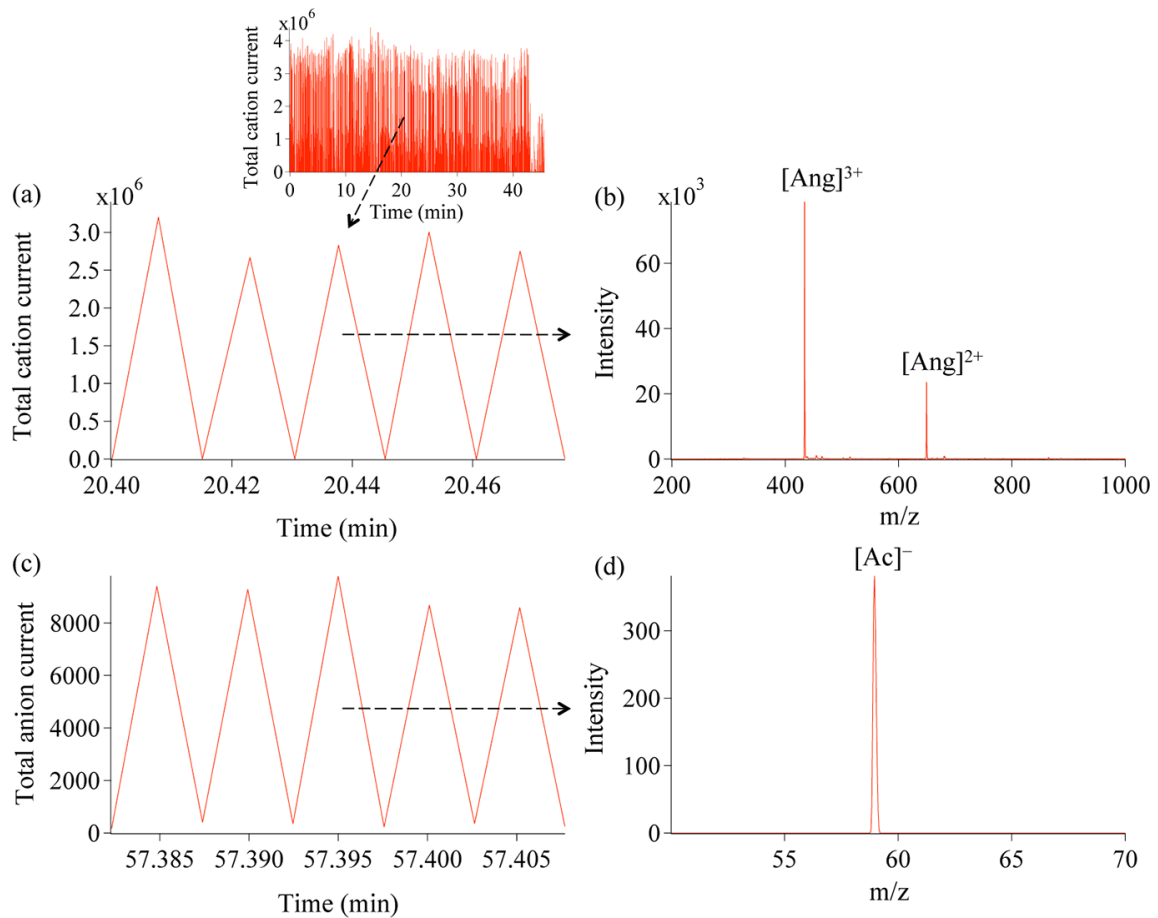

Figure 4. (a) The TCC as a function of time and (b) the mass spectrum of angiotensin I under the positive MS mode; (c) the total anion current (TAC) as a function of time and (d) the mass spectrum of acetate anions under the negative MS mode. A silica capillary was used as the emitter for ESTASI. The mass spectrometer was scanning under a range of $150 \mathrm{Th}$ to $2000 \mathrm{Th}$ ( $\mathrm{a}$ and b) or $50 \mathrm{Th}$ to $70 \mathrm{Th}(\mathrm{c}$ and d) and a scan rate of 10000 $\mathrm{Th} / \mathrm{s}$. Only a range from $200 \mathrm{Th}$ to $1000 \mathrm{Th}$ is shown in (b) since the rest is blank without any peaks.

inside the capillary, where the polymer coated silica worked as the insulator of the capacitor.

Shown as Figure 4, ESTASI was performed by infusing the angiotensin I solution $\left(0.1 \mathrm{mM}\right.$ in $50 \% \mathrm{MeOH} / 49 \% \mathrm{H}_{2} \mathrm{O} / 1 \%$ acetic acid) through the capillary at a flow rate of $20 \mu \mathrm{L} / \mathrm{h}$ and applying a high potential $(6 \mathrm{kV})$ sequence to the electrode $\left(t_{1}=\right.$ $t_{4}=0.05 \mathrm{~s}, t_{2}=0.25 \mathrm{~s}$, and $\left.t_{3}=0.15 \mathrm{~s}\right)$. When the mass spectrometer was scanned in the positive mode, angiotensin I cations were observed (Figure $4 \mathrm{~b}$ ) and the pulsed spray was found to be quite stable from the TCC response in Figure 4a. The TCC response showed a frequency of $1.1 \mathrm{~Hz}$, indicating that the generation of each spectrum took $450 \mathrm{~ms}$ and included 1.5 charging-discharging cycles.

When the mass spectrometer was scanned in the negative mode, anions generated during the capacitor discharging were detected. Under the experimental conditions, the anions were mainly acetate ions $\left([\mathrm{Ac}]^{-}, 59 \mathrm{Th}\right)$. Therefore, a $\mathrm{m} / z$ scan range of 50-70 Th was selected. Figure $4 \mathrm{c}$ shows the zoomed total anion current (TAC) as a function of time detected by the MS. The peaks of TAC appeared with a frequency of $3.33 \mathrm{~Hz}$, synchronizing the LabView program $\left(t_{1}=t_{4}=0.05 \mathrm{~s}, t_{2}=0.25\right.$ $\mathrm{s}$, and $\left.t_{3}=0.15 \mathrm{~s}\right)$. Since two spectra were collected in each cycle, the generation of each spectrum took $150 \mathrm{~ms}$ and included 0.5 charging-discharging cycles.

Figure $4 \mathrm{~d}$ shows the acetate ions detected. Deprotonated angiotensin I anions could also be observed during capacitor discharging when the angiotensin I was dissolved in a basic buffer of $20 \mathrm{mM} \mathrm{NH} \mathrm{NCO}_{3}$ in $50 \%$ water/50\% methanol, Figure SI-6, Supporting Information. The observation of both cations and anions during ESTASI induced by a positive high voltage also supports the charging-discharging principle.

During all MS experiments, an enhanced scan rate was set for the LTQ Velos, corresponding to $10000 \mathrm{Th} / \mathrm{s}$. In Figures 2 and $4 \mathrm{~b}$, the scan ranges were set to $150 \mathrm{Th}$ to $2000 \mathrm{Th}$. Therefore, the ion scanning procedure would take by itself 185 ms, already more than the half period of one chargingdischarging cycle. In Figure 4c, the scan range was $50 \mathrm{Th}$ to 70 Th, corresponding to only $2 \mathrm{~ms}$ spent for ion scanning.

In addition to ESTASI-MS analysis of peptides, the analysis of different proteins was also realized using the silica capillary as the emitter. Two proteins, myoglobin and beta-lactoglobulin ( 5 $\mu \mathrm{M}$ in $50 \% \mathrm{MeOH} / 49 \% \mathrm{H}_{2} \mathrm{O} / 1 \%$ acetic acid, respectively), were used to illustrate the principle, Figure SI-7.1, Supporting Information. This shows that ESTASI is possible to be conveniently performed after separation techniques, such as liquid chromatography (LC) and CE, where silica capillaries are usually used as the solution outlet. It should be mentioned that both negative and positive high potentials can be used to induce the ESTASI and produce cations for MS analysis benefiting from the capacitor charging-discharging principle. In contrast, MS should detect only negative ions during ESI when a negative high potential is applied on the emitter and when the MS is grounded. Even if the cations are formed during negative ESI, they will not enter the MS because of the electric field direction. In ESTASI, spray of anions happens during capacitor charging, while spray of cations happens during capacitor discharging when a negative high potential is applied on the electrode and when the MS is grounded. These ions can always enter the MS because the electric field direction is also changing during capacitor charging-discharging. Specifically, we detected myoglobin cations when a negative high potential $(-6 \mathrm{kV})$ was used to induce ESTASI and when the ion trap was scanning cations, Figure SI-7.2, Supporting Information.

ESTASI-MS from Disposable Pipet Tips. ESTASI-MS was also performed with disposable plastic pipet tips $(\sim 300 \mu \mathrm{m}$ 
inner diameter at the end), with an electrode placed around the tip to induce the spray, as shown in Scheme 1b. According to the calculation in Supporting Information SI-2, the capacitance of the first capacitor is large enough to induce electrospray. The tip was loaded with $1 \mu \mathrm{L}$ of angiotensin I solution $(0.1 \mathrm{mM}$ in $50 \% \mathrm{MeOH} / 49 \% \mathrm{H}_{2} \mathrm{O} / 1 \%$ acetic acid) and placed in front of the mass spectrometer inlet. When a high voltage $(6 \mathrm{kV})$ was applied on the electrode, cations were produced by ESTASI and detected by the mass spectrometer, Figure 5. Because of

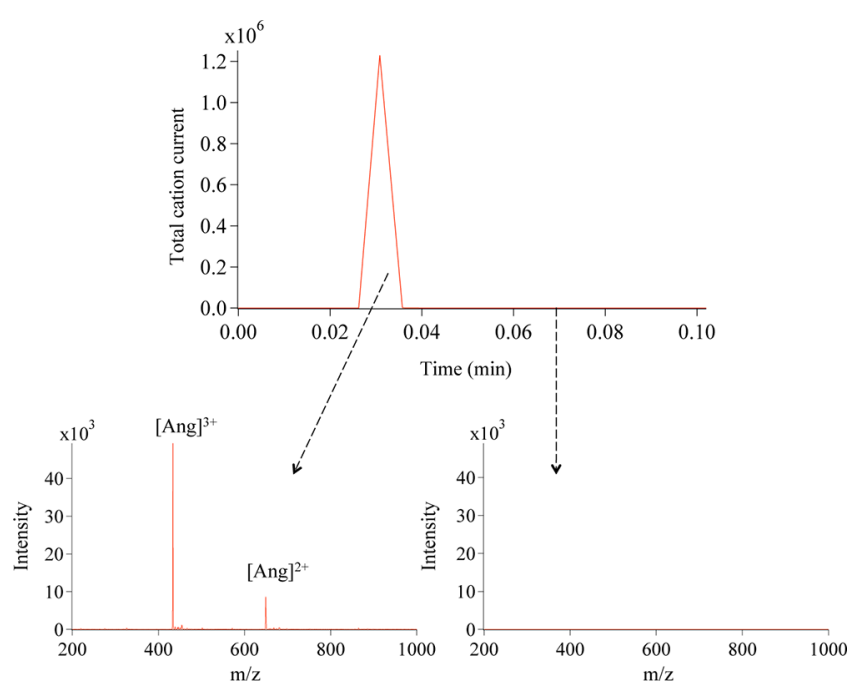

Figure 5. TCC as a function of time and mass spectrum of angiotensin I under positive MS mode. The ions for MS detection were generated by ESTASI directly from a disposable micropipet tip as illustrated in Scheme $1 \mathrm{~b}$. A positive potential of $6 \mathrm{kV}$ was used and the time sequence parameters were set to: $t_{1}=t_{4}=0.05 \mathrm{~s}, t_{2}=0.25 \mathrm{~s}$, and $t_{3}=$ $0.15 \mathrm{~s}$.

the lack of the flow and low amount of the sample, single ESTASI was generated. With the micropipet tip as emitter, it is possible to run high throughput analysis of biological samples taken directly from a microtiter plate.

ESTASI-MS from Microdroplets on an Insulating Plate. As shown in Supporting Information SI-2, microdroplets can also be sprayed by ESTASI, and the molecules in the droplets can be ionized for MS analysis. Arrays of microdroplets were deposited on an insulating plate, which was a $2 \mathrm{~mm}$-thick PMMA plate patterned with wells $(2 \mathrm{~mm}$ in diameter, $100 \mu \mathrm{m}$ deep, Scheme 1c and Figure SI-8, Supporting Information). The electrode (platinum column with the diameter of $1 \mathrm{~mm}$ ) was placed behind the PMMA plate opposite the inlet of mass spectrometer. By moving the insulating plate to bring the microdroplet close to the mass spectrometer inlet and applying pulsed high potential $(6 \mathrm{kV})$, spray of the droplet was realized and the generated ions were detected by MS.

One microliter of $0.1 \mathrm{mM}$ angiotensin I solution in $99 \%$ water and $1 \%$ acetic acid was deposited on the PMMA substrate. Singly, doubly, and triply protonated angiotensin I cations were generated by ESTASI and detected by the linear ion trap mass spectrometer, Figure 6a. Changing the droplet to $1 \mu \mathrm{L}$ of $0.05 \mathrm{mM}$ myoglobin in $99 \%$ water and $1 \%$ acetic acid, we obtained the mass spectrum of multiprotonated myoglobin ions, Figure $6 \mathrm{~b}$. Furthermore, ESTASI-MS was used to analyze a myoglobin tryptic digest $(1 \mu \mathrm{L}$ of $30 \mu \mathrm{M}$ in $50 \% \mathrm{MeOH} / 49 \%$ $\mathrm{H}_{2} \mathrm{O} / 1 \%$ acetic acid) deposited on the polymer substrate.
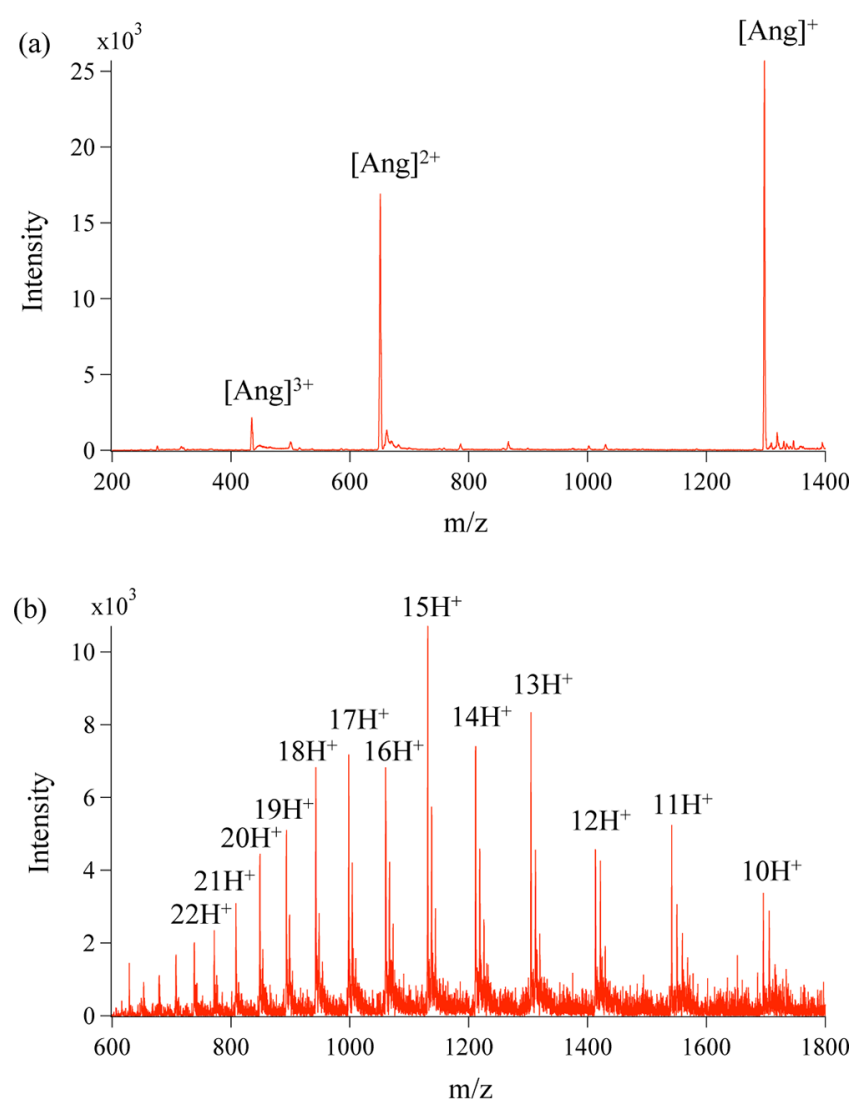

Figure 6. Mass spectra of (a) angiotensin I $\left(100 \mu \mathrm{M}\right.$ in $99 \% \mathrm{H}_{2} \mathrm{O} / 1 \%$ acetic acid) and (b) myoglobin (50 $\mu \mathrm{M}$ in $99 \% \mathrm{H}_{2} \mathrm{O} / 1 \%$ acetic acid) under positive MS mode. The ions were generated by ESTASI when a pulsed positive high potential $(6 \mathrm{kV})$ was applied to the electrode and when $1 \mu \mathrm{L}$ of the sample solution was deposited on an insulating plate. The LabView time sequence parameters were set to: $t_{1}=t_{4}=0.05 \mathrm{~s}, t_{2}$ $=0.25 \mathrm{~s}$, and $t_{3}=0.15 \mathrm{~s}$.

Shown as Figure SI-9 and Table SI-9, Supporting Information, 12 peaks were observed corresponding to 11 peptides.

This droplet spray ionization method is quite novel and has a great application potential. Since samples are deposited on a substrate as droplets for ionization, droplet ESTASI holds some similarity to matrix-assisted laser desorption/ionization (MALDI), but of course, the present approach does not require the use of a matrix nor of a laser. The present ESTASI method can be easily synchronized with pulsed time-of-flight analysis for more economical sample consumption. Combined with automatic deposition techniques, ${ }^{23}$ droplet ESTASI is able to work as an interface between separation methods, such as $\mathrm{CE}$ and LC, and MS.

As a proof-of-concept, we have employed CE to separate a mixture of peptides obtained from myoglobin digestion by trypsin. During the separation, the fractions were collected on an insulating polymer substrate for ESTASI-MS analysis with the help of a homemade robotic system that was previously designed for collecting CE fractions on a MALDI sample plate. ${ }^{23}$ The CE separation with sample collection on a plate is schematically illustrated in Scheme 3. Figure 7a shows the CEUV result of separated peptides. The peptides with the migration time between 3.5 and $8.5 \mathrm{~min}$ were collected on the polymer plate as 18 spots shown as Figure $7 \mathrm{~b}$. For comparison, the same fractions were also collected on a MALDI sample plate for MALDI MS analysis. Figure 7c,d 
Scheme 3. CE Separation with Sample Collection on a Plate

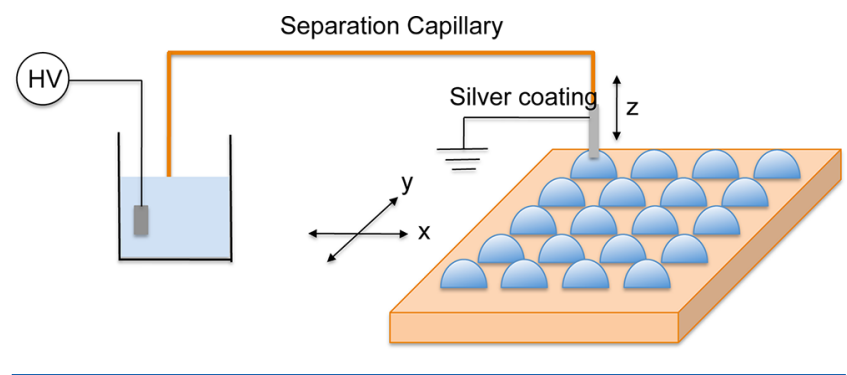

shows the mass spectra of ESTASI-MS of fractions 9 and 10, where one peptide was clearly found from each spectrum. This result is confirmed by MALDI-MS, shown as Figure SI10.1,10.2, Supporting Information. The peptides observed by ESTASI-MS and MALDI-MS from the CE fractions are compared in Table SI-10, Supporting Information. Seventeen peptides were detected by combining the two strategies from the 18 fractions. Each method identified 15 peptides, where 13 peptides were observed by both ESTASI-MS and MALDI-MS. These results indicated that the CE-ESTASI-MS strategy is as powerful as the reported CE-MALDI-MS strategy and that the two ionization strategies can be complementary.

ESTASI vS ESI. By taking advantage of the ESTASI principle, different geometries and different applications can be envisaged such as spraying from a droplet on an insulating plate. The latter opens many possibilities in analytical chemistry. With this technique, molecules on a surface, such as a piece of tissue or an electrophoresis gel, can be directly ionized for MS detection by simply adding a droplet of buffer that can dissolve the target molecule. This provides an alternative strategy to ionize a sample from a surface as compared to MALDI and desorption electrospray ionization (DESI). ${ }^{24}$ Interestingly, ESTASI can be operated on a single pulse mode, meaning that analyses can be performed consuming only a few attoliters of samples.

ESTASI-MS gave ion currents more or less as strong as ESIMS for the same concentrations of samples (protein or peptide) when emitters with similar geometries were employed, Figure SI-11, Supporting Information, Figure 2c, and Figure SI7.1a, Supporting Information. As shown in Figure 3 and Figures SI-4 and SI-5, Supporting Information, ESTASI-MS shows better LOD than ESI-MS and does not limit the detection of large proteins. During all experiments, no distinct fragmentation of peptides or proteins was observed by ESTASI compared to ESI, indicating the soft ionization nature of ESTASI. Considering that the electrode doest not contact the sample solution during ESTASI, the oxidation of samples on the electrode can be avoided; therefore, ESTASI should be better than ESI to avoid modification/fragmentation of the sample during the spray.

In addition, the ESTASI can be more economical in the view of sample consumption and MS scanning because of its pulsed spray nature. By modifying the time sequence controlling the switches and synchronizing the pulses with MS, ESTASI-MS works only when it is requested but not continuously as in conventional ESI-MS.

\section{CONCLUSION}

This work clearly shows that ESTASI is a versatile technique that offers many potential applications in analytical chemistry. The principle is based on the charging of a liquid surface with the geometry of the system that allows the focusing of the electric field to onset the formation of a Taylor cone at the surface of the liquid. From an experimental viewpoint, pulsed voltages can be applied using a DC high voltage source and a synchronized switch system. ESTASI was demonstrated on different geometries including polymer coated silica capillaries, disposable plastic micropipet tips, and polyimide microchips. The technique was further applied to spray microdroplets deposited on an insulating plate and to produce ions of the chemicals dissolved in the droplet. ESTASI is ideally suited for bioanalytical studies because of its very convenient connection with many separation techniques.

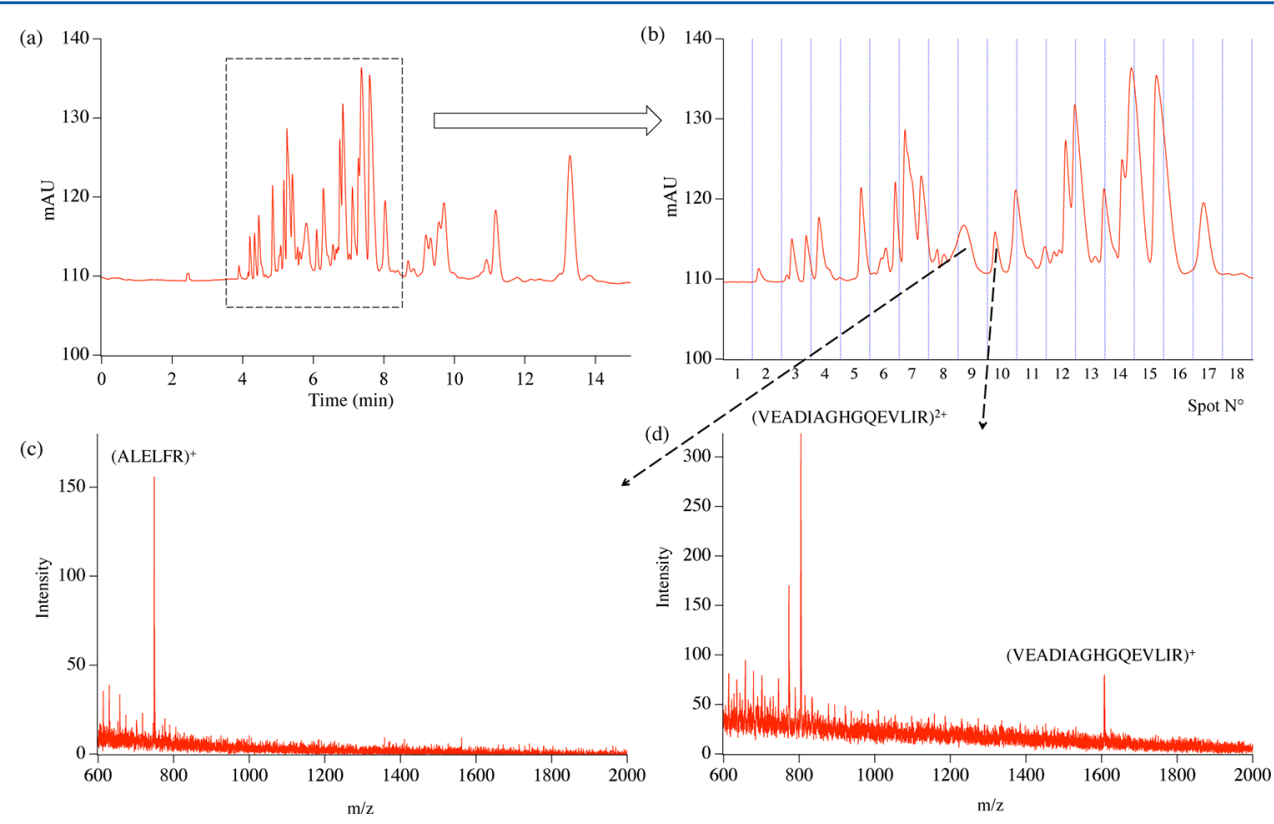

Figure 7. (a, b) CE-UV of the myoglobin tryptic digestion. (c) ESTASI-MS of fraction 9. (d) ESTASI-MS of fraction 10. 


\section{ASSOCIATED CONTENT}

\section{S Supporting Information}

(1) Chemicals and microchip fabrication, (2) ESTASI mechanism, (3) schematic illustration of the employed LabView program, (4) limit of detection of native ESI, (5) ESTASI-MS and ESI-MS analysis of large proteins, (6) ESTASI of angiotensin anions under positive voltage, (7) analysis of proteins by ESTASI-MS using a commercial silica capillary as emitter, (8) pictures of the PMMA plate patterned with wells, (9) ESTASI of a myoglobin digest deposited as droplets on the polymer plate, (10) CE-ESTASI-MS and CE-MALDI-MS, and (11) conventional ESI-MS of angiotenisn I and myoglobin. This material is available free of charge via the Internet at http://pubs.acs.org.

\section{AUTHOR INFORMATION}

\section{Corresponding Author}

*Fax: +41 (0)21 69336 67. Tel: +41 (0)21 69331 51. E-mail: hubert.girault@epfl.ch.

\section{Author Contributions}

${ }^{\S}$ L.Q. and R.S. contributed equally. The manuscript was written through contributions of all authors. All authors have given approval to the final version of the manuscript.

\section{Notes}

The authors declare no competing financial interest.

\section{ACKNOWLEDGMENTS}

We thank the Swiss National Science Foundation for supporting the project "Analytical tools for proteome analysis and redoxomics (200020-127142)".

\section{REFERENCES}

(1) Nollet, J. A. Recherches sur les causes particulières des phénomènes électriques, lère ed.; Chez les frères Guerin: Paris, 1749.

(2) Yamashita, M.; Fenn, J. B. J. Phys. Chem. 1984, 88, 4451-4459.

(3) Alexandrov, M. L.; Gall, L. N.; Krasnov, N. V.; Nikolaev, V. I.; Pavlenko, V. A.; Shkurov, V. A. Int. J. Mass Spectrom. Ion Processes 1983, 54, 231-235.

(4) Fenn, J. B.; Mann, M.; Meng, C. K.; Wong, S. F.; Whitehouse, C. M. Science 1989, 246, 64-71.

(5) Wang, Y.; Schubert, M.; Ingendoh, A.; Franzen, J. Rapid Commun. Mass Spectrom. 2000, 14, 12-17.

(6) Lu, W. Y.; Callahan, J. H.; Fry, F. S.; Andrzejewski, D.; Musser, S. M.; Harrington, P. D. Talanta 2011, 84, 1180-1187.

(7) Williams, K.; Hawkridge, A. M.; Muddiman, D. C. J. Am. Soc. Mass Spectrom. 2007, 18, 1-7.

(8) Hardman, M.; Makarov, A. A. Anal. Chem. 2003, 75, 1699-1705.

(9) Abonnenc, M.; Qiao, L. A.; Liu, B. H.; Girault, H. H. In Annual Review of Analytical Chemistry; Annual Reviews: Palo Alto, 2010; Vol. 3, pp 231-254.

(10) Rohner, T. C.; Lion, N.; Girault, H. H. Phys. Chem. Chem. Phys. 2004, 6, 3056-3068.

(11) Van Berkel, G. J.; Kertesz, V. Anal. Chem. 2007, 79, 5510-5520.

(12) Gamby, J.; Abid, J. P.; Girault, H. H. J. Am. Chem. Soc. 2005, 127, 13300-13304.

(13) Fercher, G.; Haller, A.; Smetana, W.; Vellekoopt, M. J. Anal. Chem. 2010, 82, 3270-3275.

(14) Tuma, P.; Samcova, E.; Stulik, K. Electroanalysis 2011, 23, $1870-1874$

(15) Wang, J.; Chen, G.; Muck, A. Anal. Chem. 2003, 75, 4475-4479.

(16) Huang, G.; Li, G.; Ducan, J.; Ouyang, Z.; Cooks, R. G. Angew. Chem., Int. Ed. 2011, 50, 2503-2506.

(17) Huang, G.; Li, G.; Cooks, R. G. Angew. Chem., Int. Ed. 2011, 50, 9907-9910.
(18) Peng, Y.; Zhang, S.; Gong, X.; Ma, X.; Yang, C.; Zhang, X. Anal. Chem. 2011, 83, 8863-8866.

(19) Lu, Y.; Zhou, F.; Shui, W. Q.; Bian, L. P.; Yang, P. Y. Anal. Chem. 2001, 73, 4748-4753.

(20) Chao, B. F.; Chen, C. J.; Li, F. A.; Her, G. R. Electrophoresis 2006, 27, 2083-2090.

(21) Berggren, W. T.; Westphall, M. S.; Smith, L. M. Anal. Chem. 2002, 74, 3443-3448.

(22) Wei, J. F.; Shui, W. Q.; Zhou, F.; Lu, Y.; Chen, K. K.; Xu, G. B.; Yang, P. Y. Mass Spectrom. Rev. 2002, 21, 148-162.

(23) Busnel, J.-M.; Josserand, J.; Lion, N.; Girault, H. H. Anal. Chem. 2009, 81, 3867-3872.

(24) Takats, Z.; Wiseman, J. M.; Gologan, B.; Cooks, R. G. Science 2004, 306, 471-473. 